Colloque C2, suppl. au Journal de Physique II, Vol. 1, septembre 1991

\title{
EPITAXIAL SILICON GROWTH BY RAPID THERMAL CVD
}

\author{
D.W. MCNEILL, Y. LIANG, J.H. MONTGOMERY, H.S. GAMBLE and \\ B.M. ARMSTRONG
}

\begin{abstract}
Institute of Advanced Microelectronics, Department of Electrical and Electronic Engineering, The Queen's University of Belfast, Ashby Building, Stranmillis Road, IR-Belfast Br9 $5 A H$, Northern Ireland
\end{abstract}

\begin{abstract}
A rapid thermal chemical vapour deposition system has been used for the growth of epitaxial silicon layers on silicon substrates in the temperature range $590^{\circ} \mathrm{C}-1000^{\circ} \mathrm{C}$. Low pressure deposition schedules have been found essential in order to minimise the concentrations of oxygen and carbon in the layers. In-situ pre-cleaning schedules have been established to remove oxide layers from the substrates prior to layer deposition. The application of these deposited epitaxial layers to the production of a buried gate static induction thyristor is described.
\end{abstract}

\section{INTRODUCTIOW}

The deposition of polycrystalline silicon and the growth of epitaxial silicon are vital steps in advanced integrated circuit processes. The technique in almost universal use for the production of these films is chemical vapour deposition (CVD), wherein gaseous species (eg $\mathrm{SiH}_{4}, \mathrm{SiH}_{2} \mathrm{Cl}_{2}$ ) react on a substrate and a solid phase material (silicon) is produced. Both polysilicon and epitaxial silicon have traditionally been produced in atmospheric pressure reactors. However, in the case of polysilicon, low pressure reactors have largely taken over because of improved uniformity and reduced gas consumption. Reduced pressures are also beneficial for the growth of epitaxial silicon, as autodoping and pattern shift effects are alleviated [1] and homogeneous gas phase nucleation of silane is retarded [2]. Low pressure (LP) CVD is thus, becoming the most technologically important means of producing silicon films.

Recently, much attention has been given to a refinement of LPCVD which incorporates the concept of rapid thermal processing (RTP). In RTP, the single process wafer is heated by a high-intensity light source, and very fast ramp rates can be achieved. The combination of LPCVD and RTP is known as rapid thermal chemical vapour deposition (RTCVD). In particular, when reactions are triggered by a temperature pulse (rather than by gas switching), the technique has been termed limited reaction processing (LRP) [3].

Rapid thermal processes have the advantage of a reduced thermal budget, as the fast ramp rates minimize the time for which the wafer is at elevated temperatures. This is an important consideration for high-speed VLSI circuitry, where lateral and vertical device dimensions must be tightly controlled. Because RTP minimizes unwanted dopant diffusion, shallow junctions of high quality are more readily obtained by this technique than by traditional furnace methods. The ability to switch temperatures rapidly also means that several different process steps can be carried out sequentially in-situ. 
LRP shares the advantages of RTP, and may be used to deposit a sequence of thin, highly abrupt semiconducting layers. Applications include shallow emitter-base structures for heterojunction bipolar transistors [4, 5] and superlattices [6], and buried $\mathrm{p}^{+}$grid structures for advanced static induction thyristors.

This paper will describe a twin reactor system used for the production of epitaxial silicon layers for device manufacture. One of the reactors has been dedicated to high temperature epitaxy from $\mathrm{SiH}_{2} \mathrm{Cl}_{2}$ and the other to low temperature epitaxy from $\mathrm{SiH}_{4}$. The requirements and properties of the two processes will be contrasted.

\section{THE QUPLAS REACTOR}

The systems designed for this work are referred to as QUPLAS reactors. They are designed for single wafer processing and are currently able to handle 2" substrates. A simplified cross-section of the reactor is shown in Figure 1 . The reaction chamber is a horizontal quartz tube with a rectangular cross-section of area $40 \mathrm{~cm}^{2}$ and total volume of 4 litres. The process wafer is supported by four quartz pins on a quartz boat. The boat is attached to the loading door which has an "O"-ring seal. This ensures that successive wafers are located in an identical position in the chamber.

Process gases are introduced at the opposite end of the chamber from both the loading door and the vacuum port. Up to six gases may be separately selected and controlled by mass flow controllers. These are then mixed in a gas manifold prior to entering the reactor.

Wafer heating is achieved using banks of tungsten halogen lamps. For the low temperature epitaxy the banks are placed above and below the wafer and each contain nine $0.5 \mathrm{~kW}$ lamps. The higher temperature epitaxy is undertaken with two banks of lamps with a total input power of $18 \mathrm{~kW}$. The quartz chamber walls are essentially transparent to the light emitted from the lamps, and thus only the wafer reaches the process temperature. However, the quartz will gradually heat up due to re-radiation from the wafer and some cooling is necessary. Forced air is used to cool both the outside of the chamber walls and the lamp-banks. This is supplemented by jets of high pressure air during higher temperature processing ( $850^{\circ} \mathrm{C}$ ).

A simple two-stage mechanical rotary pump is employed to evacuate the reaction

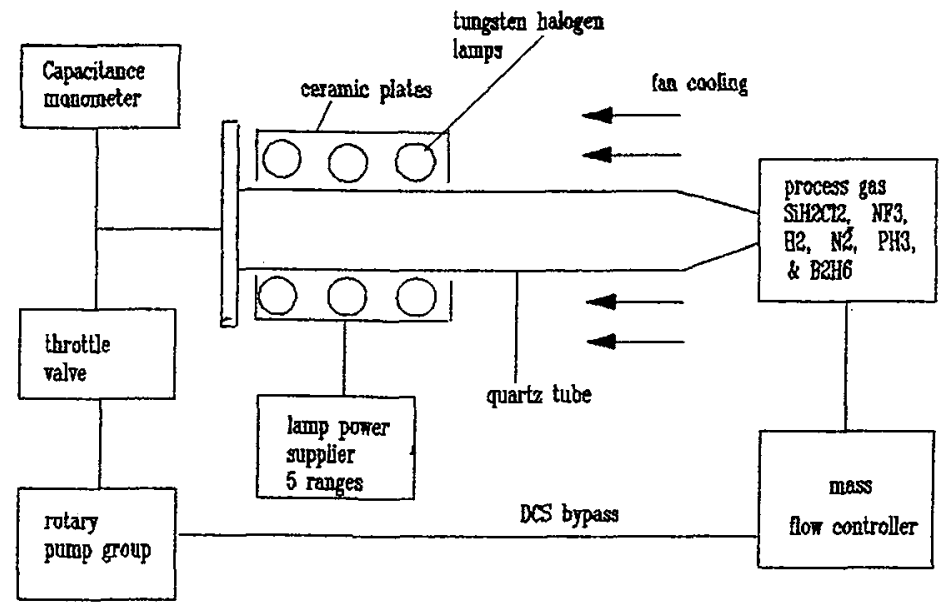

Fig. I QUPLAS reactor schematic 
chamber for the higher temperature epitaxial silicon deposition. The depositions at lower temperature are more susceptible to residual oxygen and water vapour in the environment. A rotary and turbomolecular pump combination capable of base pressures of the order $10^{-7}-10^{-6}$ mbar has therefore been used on this reactor.

Input power is controlled by a pulse-width modulator, which operates with a $100 \mathrm{~ms}$ period. Power settings are converted to a control pulse of proportional time duration. However, because the relays which control the lamps only operate on positive zero-crossings of the mains voltage, only a limited number of discrete pulse widths are possible. With three phases, and a $20 \mathrm{~ms}$ mains period, 15 different temperatures can be obtained, corresponding to different pulse widths. Some typical temperature-time profiles are shown in $F$ ig. 2 .

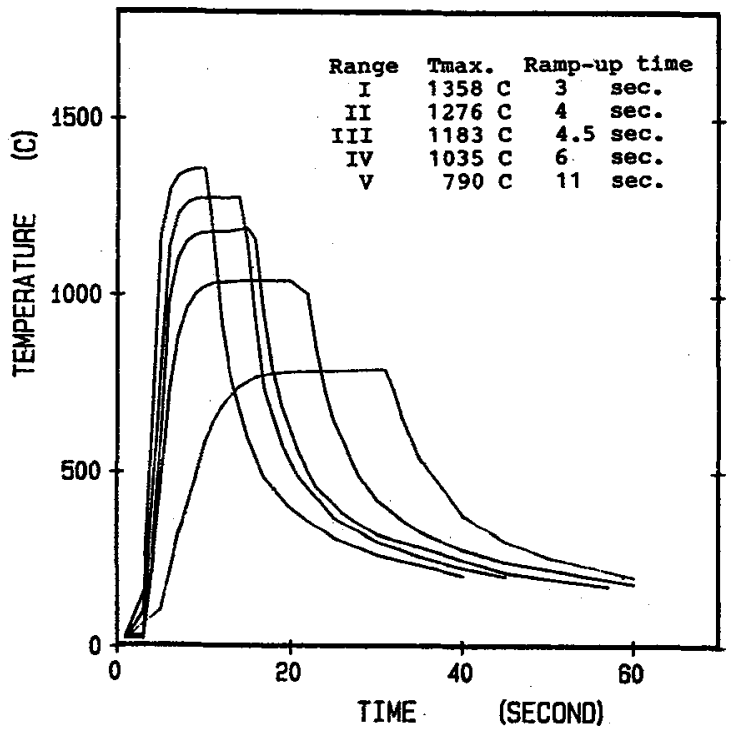

Fig.2 Temperature - time profiles

\section{SILICON DEPOSITION AT HIGH TEMPERATURE}

Device quality epitaxial layers of silicon are more readily achieved at high temperature. In this work the gas employed was $\mathrm{SiH}_{2} \mathrm{Cl}_{2}$ with hydrogen as an additional carrier gas. Initial work was in the LRP regime with the gas flow established and the deposition process triggered by the temperature pulse. Substrates received a standard wet chemical clean and a modified RCA clean to produce a hydrous oxide film [7]. Depositions were carried out at a temperature of $1035^{\circ} \mathrm{C}$ and at a pressure of 10 Torr with hydrogen flow $1000 \mathrm{sccm}$ and a dichlorosilane flow of $29 \mathrm{sccm}$. The deposition rate under these conditions is typically $0.6 \mu \mathrm{m}$ per minute. The ramp up time from ambient to process temperature was six seconds and it was hoped that the surface oxide layer would desorb during this time period. This did not however occur and the layers exhibited large oxygen ard carbon concentrations at the interface and substantial carbon concentrations in the deposited layer. The ramp up period was clearly too short to permit desorption of the oxide layer. The process was modified to include an initial hydrogen bake for 1 minute at $1035^{\circ} \mathrm{C}$ followed after rapid cool down and establishment of the $\mathrm{SiH}_{2} \mathrm{Cl}_{2}$ flow rate by the deposition step. A typical SIMS profile of the resultant layer is shown in Fig.3(a). Oxygen and carbon concentrations in the deposited layer have been reduced but there are still substantial peak values of these impurities at the growth interface. Clearly the oxide layer regrows during the time that the wafer is at low temperature between the pre-clean and deposition time 
pexiods. This shows a disadvantage of the LRP regime. The system has therefore been modified to permit gas switching during the time at high temperature yielding an RTCVD process. Precleaning is achieved in the hydrogen ambient and then the $\mathrm{SiH}_{2} \mathrm{Cl}_{2}$ is switched into the reactor to ensure deposition on an oxide free surface. The SIMS profile of the layer is shown in Fig.3(b). The oxide interface is completely removed but the peak concentration in the carbon is only marginally reduced. The precise reason for this carbon peak is not clear but may be due to backstreaming from the rotary pump.

Electrical characterisation has been undertaken on these layers with the manufacture of $\mathbf{p}-\mathbf{n}$ diodes and MOS capacitors. The Zerbst analysis techniques yield typical lifetimes for these layers of approximately $200 \mu \mathrm{s}$ and carrier concentrations of electrons 5-7 $\times 10^{14} \mathrm{~cm}^{-3}$. Diodes exhibited good current-voltage characteristics with typical reverse leakage current densities of $3 \mathrm{nA}^{\mathrm{cm}} \mathrm{cm}^{-2}$.

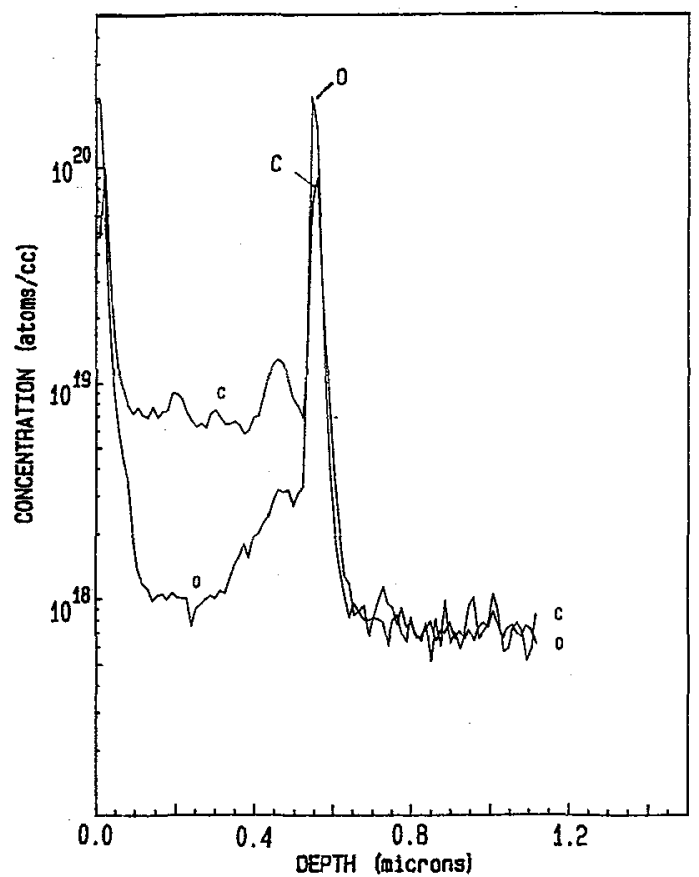

(a)

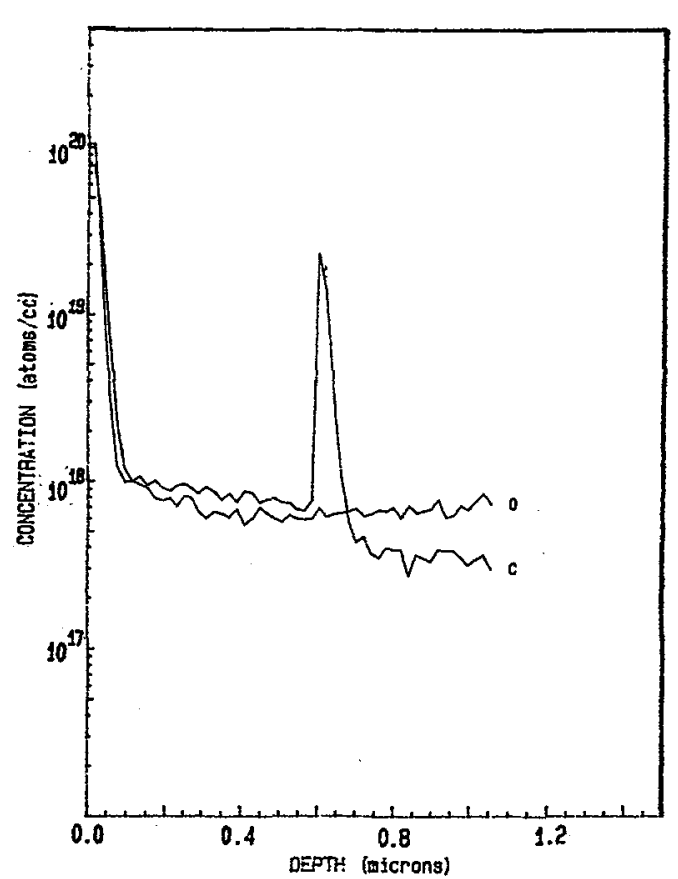

(b)

Fig.3 SIMS profiles of silicon deposited by (a) LRP (b) RTCVD

A major advantage of the RTCVD regime is the reduced thermal budget. This is clearly illustrated by the growth of the epitaxial layer on a sample wafer which has been previously doped with boron. The SIMS profile of Fig.4(a) shows the very steep transition in doping profile down to the sIMS background level of detection, with two orders of magnitude reduction in boron concentration over a distance of 30 nm.

A further advantage of the RTCVD regime is in the avoidance of autodoping effects. This can be a serious problem when the epitaxial layer has to be deposited on a substrate which has heavily doped patterned regions exposed on the surface. The problem has been highlighted in this work with the production of a Buried Grid Static Induction Thyristor. (SI Thy), which offers the promise of a high power, high frequency switching device with full gate control. The basic structure is shown in Fig.4(b). The buried grid gate controls the operation of the device and 
consists of heavily diffused boron regions. The device structure can only be fabricated by diffusion of the grid into the high resistivity wafer surface ( $600 \Omega-c m n$ type), followed by deposition of the undoped epitaxial silicon layer into which the cathode is formed. If a conventional epitaxial reactor were used in the production of this device, the wafer would be at the process temperature for a considerable time prior to the introduction of the process gases. During this period boron will evaporate from the $\mathrm{P}^{+}$grid into the boundary layer above the wafer. Thus when the deposition gas is introduced, the first layers of epitaxial growth will be doped with boron from the boundary layer, with the level depending on the prebake and growth conditions used. This means that initially a continuous p-gate layer is formed during deposition of the undoped epitaxial layer $\left(5 \times 10^{14} \mathrm{~cm}^{-3}\right)$, resulting in failure of the device.

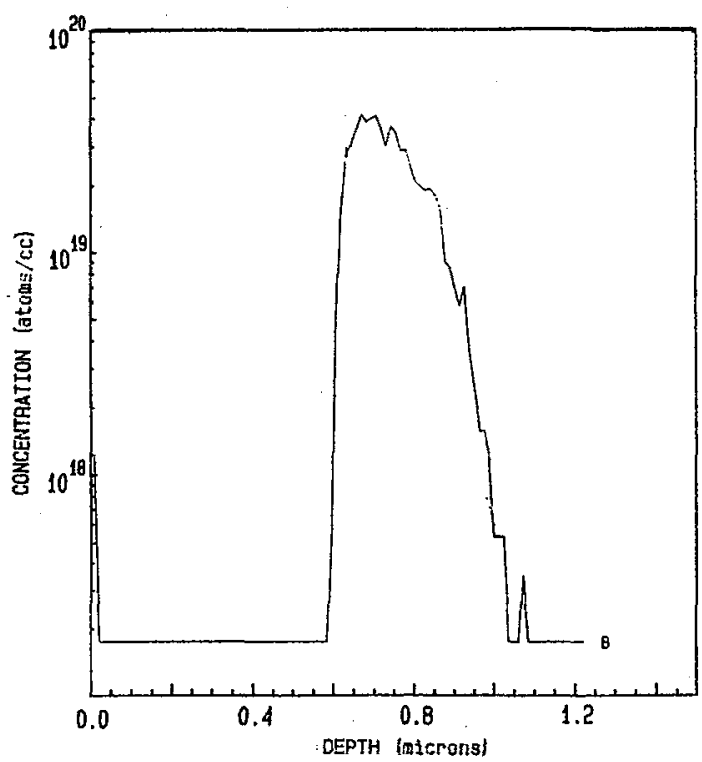

(a)

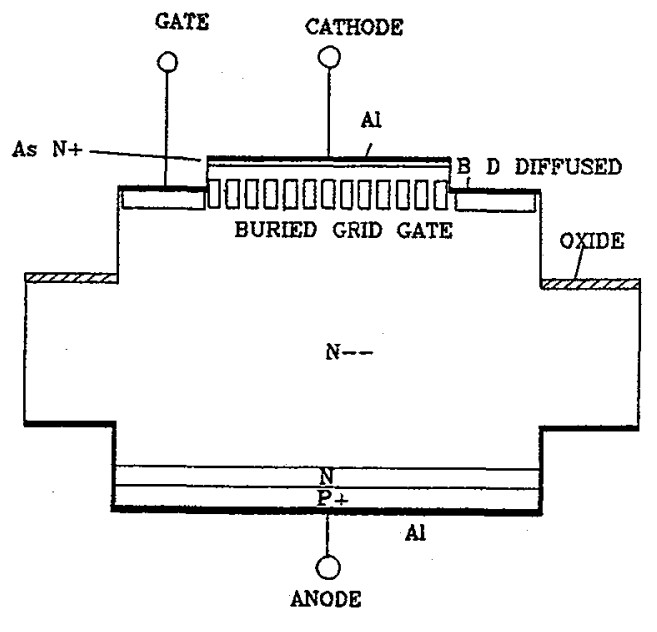

(b)

Fig.4(a) SIMS profile of an undoped silicon layer deposited on a heavily doped substrate

(b) Cross-section schematic of the buried grid SI Thy

The RTCVD regime previously described has been used in the production of these devices. The hydrogen pre-bake time is optimised to ensure the native oxide on the process wafer is just removed, and the $\mathrm{SiH}_{2} \mathrm{Cl}_{2}$ is then switched in to deposit the layer before boron evaporation from the surface can take place. A cross-section optical micrograph through the structure is shown in Fig.5(a). The $P^{+}$silicon fingers in the grid have been chemically stained and appear as dark regions. The light region surrounding each finger is the depletion layer associated with the $\mathrm{P}^{+} \mathrm{N}$ junction. It is clear from the photograph that autodoping has not occurred. There is no evidence of any p-type region connecting the grid fingers together. The completed devices operated satisfactorily. A typical switching characteristic for the SI.Thy is shown in Fig.5(b). The current flow between cathode and anode is displayed against the applied anode voltage for several values of grid voltage. clear modulation of the blocking voltage of the device and the on state current is observed under the control of the buried grid.

Device quality epitaxial silicon layers have been achieved using this relatively simple RTCVD system. There are many potential applications for the system in the production of advanced solid state devices and circuits. There is however a need 
for such layers to be deposited at lower process temperatures and the need for the development of a system suited to the deposition of other materials e.g. Si:Ge at lower temperatures. The second chamber of this system addresses these areas.

\section{SILICON DEPOSITION AT LONER TEMPERATURES}

The main aim of this work has been to achieve epitaxial silicon layer deposition at temperatures of $750^{\circ} \mathrm{C}$ and below. The depositions were carried out using $20 \%$ silane diluted in nitrogen usually without an additional flow of hydrogen. At these lower temperatures the layers are more susceptible to contamination particularly from oxygen and carbon. This reactor had mechanical and turbomolecular pumps capable of achieving a base pressure of $10^{-7}$ mbar. Epitaxial silicon layers have been deposited at temperatures down to $590^{\circ} \mathrm{C}$. An electron diffraction pattern for a layer deposited at this temperature is shown in Fig.6(a). Oxygen contamination in

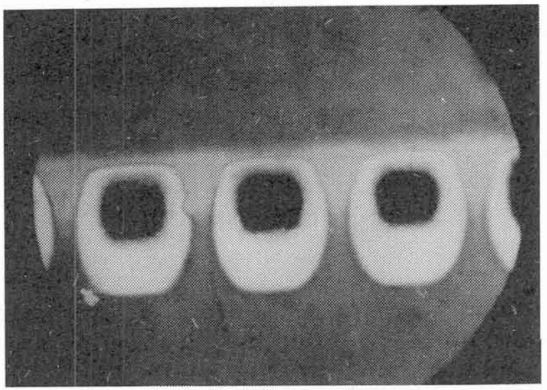

(a)

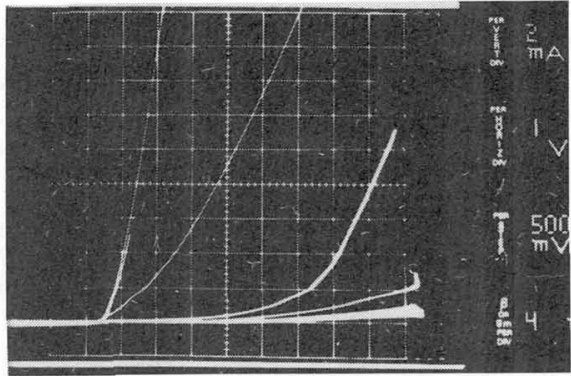

(b)

Fig.5(a) Cross-section optical micrograph of the $\mathrm{P}^{+}$grid in the SI Thy 5(b) Typical switching characteristics of the SI Thy.

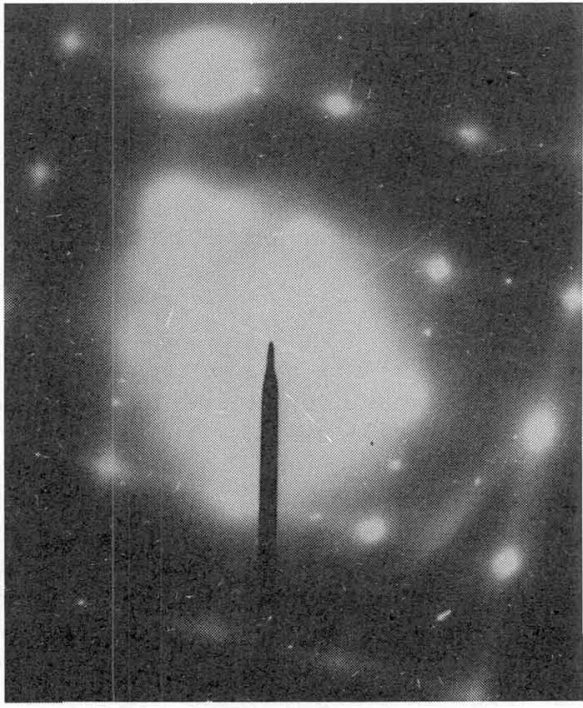

(a)

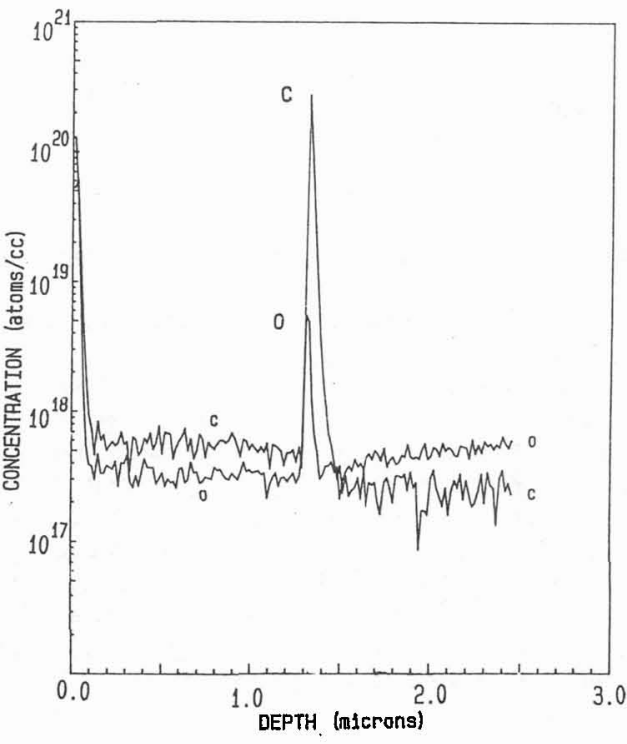

(b)

Fig. 6(a) Electron diffraction pattern for a silicon layer deposited at $590^{\circ} \mathrm{C}$ 6(b) SIMS profile for a silicon epitaxial layer deposited at $720^{\circ} \mathrm{C}$ and 0.07 mbar 
the layers has been minimised using point of source Nanochem purifiers in the gas lines. This reduces contamination in the gas to $10 \mathrm{ppb}$ but it is still essential to deposit the films at low pressure in order to maintain system pumping speed and hence keep the water vapour partial pressure below the critical value which will cause oxygen to be incorporated in the layers[8]. A typical sIMs profile of a layer deposited at 0.07 mbar and $720^{\circ} \mathrm{C}$ is shown in Fig.6(b). The concentration of oxygen and carbon impurities in the layer is clearly below the resolution limits of the SIMS. The influence of process pressure on the concentrations of these impurities is shown in Fig.7(a). A sharp rise in impurity concentration is observed for pressures greater than 0.5 mbar. At this pressure, pumping speed is sufficiently degraded to cause the water vapour partial pressure to rise above the critical value necessary for oxide formation. This upper limit on process pressure decreases as the process temperature is lowered and represents a fundamental system limitation to deposition processes in the low temperature regime. To date layers with oxygen levels less than the SIMS detection limit $\left(3 \times 10^{17} \mathrm{~cm}^{-3}\right.$ ) have been deposited at temperatures down to $720^{\circ} \mathrm{C}$. The addition of a load lock on the reactor will therefore be necessary for lower temperature work.

As with the work at high temperature, the quality of the epitaxial film is strongly dependant on the pre-cleaning of the silicon substrate. For applications where low temperature epitaxy is required it would be desireable to employ low temperature cleaning processes. The QUPLAS reactor has an upstream microwave cavity capable of creating a gas plasma within the reactor tube. $\mathrm{CF}_{4}$ plasma pre-cleaning at room temperature has been employed to remove the hydrous oxide layer from the substrate. This can usually be achieved with a 10 second process and has been employed successfully in the production of bipolar transistors [9] and thin film transistors [10]. However a drawback of the technique is the carbon contamination of the surface caused by the $\mathrm{CF}_{4}$ Hydrogen plasma cleaning at room temperature and process temperature has also been employed but found to be less effective in the removal of the oxide layer. Later work will employ either NF or HF in-situ cleaning at low temperature.

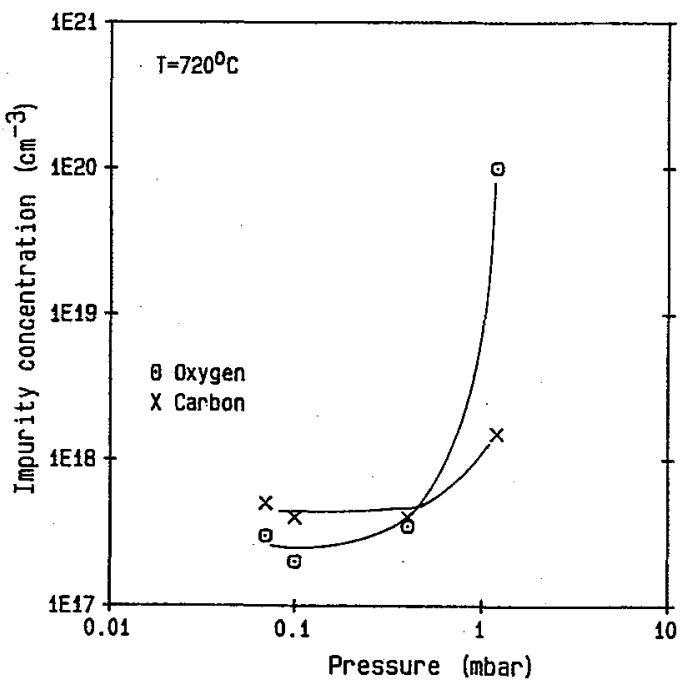

(a)

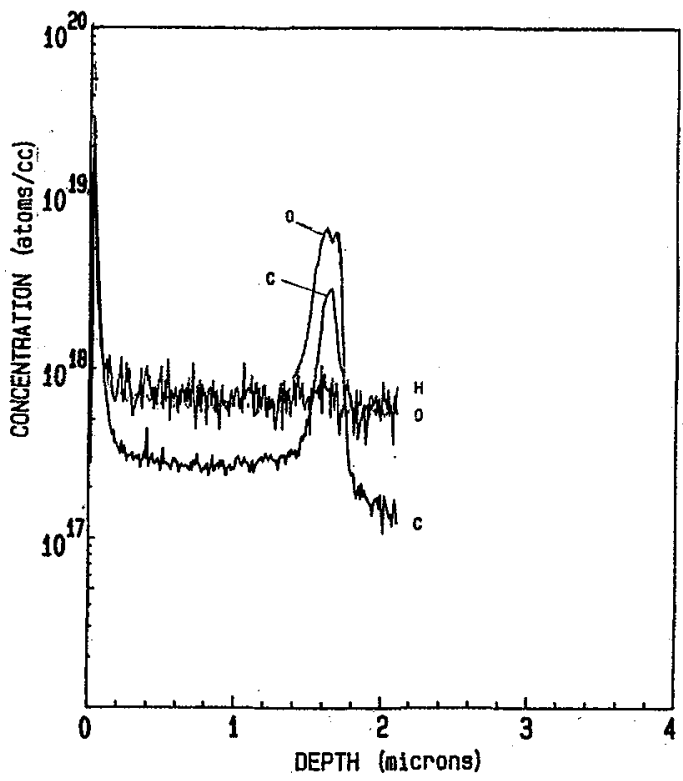

(b)

Fig.7(a) Impurity concentration against process pressure, $720^{\circ} \mathrm{C}$ $7(b)$ SIMS profile of silicon deposited after optimun pre-clean 
A short time heat pulse at high temperature in hydrogen gas has been found to be suitable in reducing interface oxygen and carbon concentrations. Typically the oxide layer is removed by a five minute in-situ bake in hydrogen at $975^{\circ} \mathrm{C}$. It is important that during the cool down to the deposition temperature the hydrogen flow is maintained without compromising pumping speed and water vapour partial pressure. In this way the doses of interfacial oxygen and carbon, as measured by integration of the SIMS depth profiles, have been reduced to $6 \times 10^{13} \mathrm{~cm}^{-2}$ and $5 \times 10^{13} \mathrm{~cm}^{-2}$ respectively. A typical SIMS plot of a layer deposited after this pre-clean is shown in Fig.7(b). The electrical quality of these layers has been examined using MOS capacitors. Zerbst plots on layers deposited at $720^{\circ} \mathrm{C}$ have shown typical carrier lifetimes of Several microseconds and carrier concentrations of the order $10^{15} \mathrm{~cm}^{-3}$.

\section{CONCLUSIONS}

A relatively simple reactor has been employed for the RTCVD of silicon. Device quality layers have been deposited at a temperature of $1000^{\circ} \mathrm{C}$ with minimised out diffusion and autodoping effects. The layers have been used for the production of a buried gate static induction thyristor. Epitaxial alignment of the deposited layers has been observed at process temperatures as $10 \mathrm{w}$ as $590^{\circ} \mathrm{C}$. However, layers with oxygen content lower than the SIMS detection limit have only been observed at deposition temperatures of $720^{\circ} \mathrm{C}$ and above. Hydrogen baking at $975^{\circ} \mathrm{C}$ has been successful in minimizing the interfacial oxygen and carbon contamination. Layers produced at $720^{\circ} \mathrm{C}$ have been of device quality with carrier lifetimes of several microseconds.

\section{ACKNONLEDGEMENTS}

The authors wish to acknowledge the financial support of the Science and Engineering Research Council and the International Fund for Ireland.

\section{REFERENCES}

[1] Herring R.B., Solid State Technol., 22 (1979), 75.

[2] Qian Z.M. et al, J. Electrochem Soc,, 135 (1988), 2378

[3] Gibbons J.F. et al, Appl. Phys, Lett. 47 (1985), 721.

[4] Green M.L. et al, Mat, Res. Soc, Symp. Proc., 146 (1989), 55

[5] King C.A. et al, Mat. Res, Soc, Symp. Proc., 146 (1989), 71.

[6] Gronet C.M. et al, J. Appl. Phys., 61 (1987), 2407.

[7] Burns G.P. \& Wilkes J.G., Semicon Sci. Technol., 3 (1988) 442

[8] Meyerson B.S. et al, J. Electrochem. Soc., 133b (1986) 1232

[9] Ruddell F, et al, Semicon Sci. Technol., 5 (1990) 765.

[10] Mitchell N.S.J. et al, Mat. Res. Soc. Symp. Proc., 182 (1990) 35. 